\title{
Gradhiva
}

GRADHI

Revue d'anthropologie et d'histoire des arts

$7 \mid 2008$

Le possédé spectaculaire

\section{La collecte comme iconoclasme}

La London Missionary Society en Polynésie

Collecting as Iconoclasm. The London Missionary Society in Polynesia

\section{Steven Hooper}

Traducteur : Ludovic Coupaye

\section{OpenEdition}

\section{Journals}

Édition électronique

URL : http://journals.openedition.org/gradhiva/1101

DOI : 10.4000/gradhiva. 1101

ISSN : 1760-849X

\section{Éditeur}

Musée du quai Branly Jacques Chirac

\section{Édition imprimée}

Date de publication : 15 mai 2008

Pagination : 120-133

ISBN : 978-2-915133-86-8

ISSN : 0764-8928

Référence électronique

Steven Hooper, «La collecte comme iconoclasme», Gradhiva [En ligne], 7 | 2008, mis en ligne le 15 mai 2011, consulté le 04 mai 2019. URL : http://journals.openedition.org/gradhiva/1101 ; DOI : 10.4000/gradhiva.1101 


\section{THE FAMULY IDOLS OF POMARE,}

Which he relinquished, and sent to the Missionaries at Eimeo, either to be burnt, or sent to the Society.

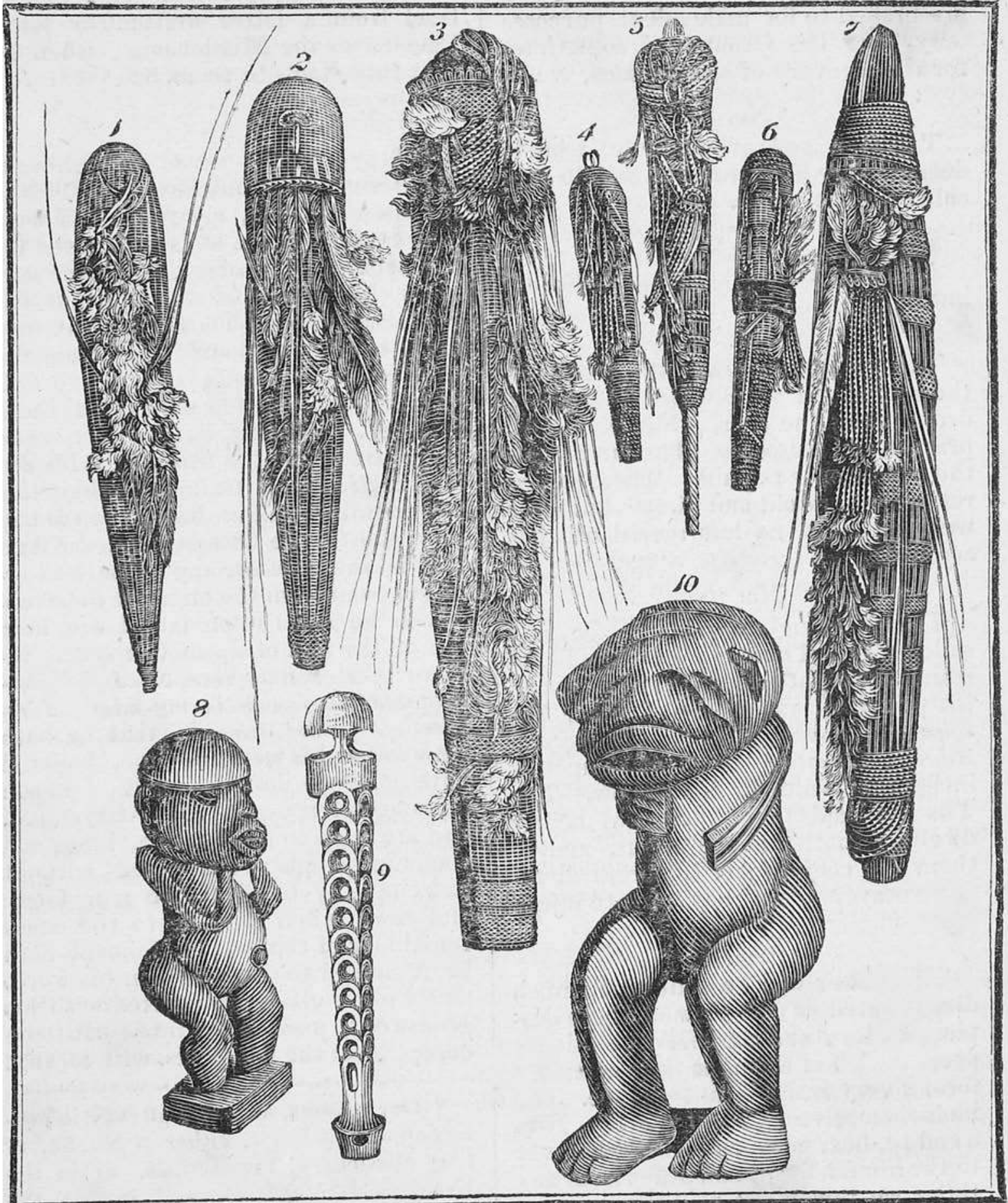

Fig. 1 Les Idoles familiales de Pomaré, Missionary Sketches III, 1818. D’après les archives de la London Missionary Society/ Council for World Mission, Londres. Photo Glenn Ratcliffe. 


\title{
La collecte comme iconoclasme
}

\author{
La London Missionary Society en Polynésie
}

\section{Steven Hooper}

\begin{abstract}
"Si vous le jugez approprié, vous pouvez les brûler toutes; ou, si vous le voulez, envoyez-les dans votre pays pour être inspectées par les peuples d'Europe afin qu'ils puissent satisfaire leur curiosité et connaître les dieux ineptes de Tahiti. »
\end{abstract}

(Pomaré, un chef de Tahiti, 1816)

L' analyse des circonstances spécifiques de l'acquisition des objets de Polynésie entre la fin du $\mathrm{XVIII}^{\mathrm{e}}$ et le début du XIX $\mathrm{x}^{\mathrm{e}}$ siècle peut nous en apprendre beaucoup sur les relations interculturelles et les processus historiques de cette époque. Le terme « appropriation " ne suffit pas à transcrire la complexité de ces relations et de ces processus, car les contextes dans lesquels ces acquisitions eurent lieu ont été ceux de stratégies d'échanges, où indigènes comme visiteurs s'offraient mutuellement des cadeaux, pratiquaient le troc ou commettaient des vols ${ }^{1}$. Chaque camp cherchait à acquérir un avantage tactique dans des situations fluides et imprévisibles; aussi un examen des motivations des personnes impliquées dans ces transactions d'objets nous permet de mieux comprendre la nature de ces échanges et la valeur historique des œuvres d'art polynésiennes qui ont survécu jusqu'à nous.

Cet article concerne principalement plusieurs objets religieux importants acquis par des membres de la London Missionary Society (LMS) en Polynésie durant les années 1820 et 1830 . Ces objets sont aujourd'hui conservés au British Museum, à Londres, aux côtés d'importantes collections polynésiennes issues d'expéditions dirigées par Samuel Wallis, James Cook et George
Vancouver au XVIII siècle $^{2}$. La LMS, fondée sous le nom de Missionary Society en 1795 et qui changea de nom en 1818, était une organisation protestante - sans restriction de dénomination - qui propagea l'Évangile dans de nombreuses régions du monde. Les premières missions en Polynésie s'établirent en 1797 aux îles de la Société, aux Tonga et aux îles Marquises, mais elles ne rencontrèrent initialement qu'un faible succès. Les missions des Tonga et des Marquises furent abandonnées, mais un avant-poste fut maintenu aux îles de la Société. Dès le début des années 1820, les missions basées à Tahiti, Mo'orea, Huahine et Ra'iatea commencèrent à exercer une influence majeure sur la religion

1. Ces trois modes de transaction rappellent le modèle proposé par Marshall Sahlins (1974) des trois formes de réciprocité : généralisée, équilibrée ou négative. Le troc, appelé trade par les premiers voyageurs, a été discuté sous une perspective anthropologique dans Humphreys et Hugh-Jones (1992). Le terme «appropriation » a été utilisé comme catégorie analytique efficace par, entre autres, Borofsky et Howard (1989: 250 et suiv., qui analyse également le vol) et par Thomas (1991 : 151-157), qui ont également étudié les collectes des missionnaires.

2. Plusieurs objets du British Museum et d'autres collections ont été rassemblés pour des expositions temporaires : Pacific Encounters au Sainsbury Centre à Norwich (Hooper 2006) et Polynésie : arts et divinités 1760-1860 au musée du quai Branly (Hooper 2008). 
et la politique locales. Les deux chefs les plus puissants de la région, Pomaré de Tahiti et Tamatoa de Ra'iatea, s'étaient alors convertis au christianisme, suivis par nombre de leurs sujets. Certaines communautés rivales leur résistèrent pendant quelque temps, puis ces groupes chrétiens alliés aux missionnaires, anglais d'abord et français par la suite, finirent par établir leur domination.

L'importance des missionnaires de la LMS pour notre propos réside en ce qu'ils s'intéressaient non seulement aux conversions et aux baptêmes, mais en ce qu'ils commencèrent également à rassembler des témoins matériels de leurs succès - preuves qu'ils pouvaient envoyer en Angleterre. Ces témoins prirent la forme d' « idoles » et autres objets rituels qui leur avaient été remis par les Polynésiens comme preuve de leur conversion au christianisme3. Ces objets, vus par les missionnaires comme les trophées de leurs batailles contre le paganisme, ont survécu jusqu'à aujourd'hui. Ceux qui se trouvent au British Museum furent prêtés en dépôt par le musée de la LMS en 1890 et officiellement acquis en 1911. D'autres musées et organisations reçurent également des objets au cours du xIx ${ }^{e}$ siècle par l'entremise de missionnaires et de leurs associés, tel George Bennet, qui dirigea avec le révérend Daniel Tyerman une tournée d'inspection des missions de la LMS à travers le monde entre 1821 et 1829, rassemblant ainsi une large collection qui, par la suite, fut répartie en de nombreux endroits en Angleterre ${ }^{4}$.

\section{La collecte comme forme d'iconoclasme}

Généralement, le but premier d'une collecte est celui de la préservation, et de manière concomitante le contrôle exercé sur ce qui est préservé. La collecte des idoles et l'iconoclasme peuvent ainsi apparaître comme des activités incompatibles - la première orientée vers la préservation et la seconde vers la destruction. Pourtant, il existe fréquemment chez les iconoclastes de fortes motivations à vouloir préserver la preuve de leur travail de destruction, notamment comme preuve de désacralisation, d'humiliation et de triomphe. Dans le cas des missionnaires, la collecte comme forme d'iconoclasme, correspondant à la logique de l'époque, a créé un paradoxe lorsque les trophées de l'évangélisation $\mathrm{du}$ xIx ${ }^{\mathrm{e}}$ siècle se sont ironiquement transformés, au xxi ${ }^{e}$ siècle, en œuvres d'art réévaluées comme héritage et objets sacrés. La survie physique de ces objets leur a ainsi permis de continuer à exercer une influence et une agency ${ }^{5}$ de manière inattendue et imprévisible.
Que les missionnaires de la LMS et leur convertis polynésiens aient été des iconoclastes au sens conventionnel du terme n'est pas discutable, car on ne peut douter du nombre important d'objets rituels qui furent détruits dans toute la Polynésie ${ }^{6}$. Cependant, la survie d'un nombre important d'images de Polynésie centrale, au moment de cette destruction, démontre que ces missionnaires étaient également soucieux d'obtenir des trophées. On retrouve cette préoccupation d'une manière explicite dans les textes de missionnaires tel que John Williams, qui relate ainsi son arrivée à Ra'iatea en 1823 , lors de son retour d'Aiutaki, à bord du navire de la mission : "Comme certains guerriers éprouvent de la fierté à exposer les trophées des victoires remportées, nous suspendîmes les idoles aux taquets des vergues et aux autres parties du vaisseau, entrâmes au port triomphalement, longeant la colonie, et jetâmes l'ancre au milieu des cris et des félicitations de notre peuple » (Williams 1837 : 106-107).

Cette exposition publique des idoles à bord du navire, et plus tard dans la chapelle de Ra'iatea, était un acte

3. Le sens donné ici au terme « idole » n'est pas péjoratif et suit la défi-
nition donnée par Gell ( 1998 : 96 et suiv.], qui analyse ces objets
comme étant le centre d'activités rituelles et une source d'« intention-
nalité » [NdT : agency, cf. note 5].
4. Voir Jacobs (à paraître). Quelques-uns des objets de George Bennet
sont retournés en Polynésie, notamment quatre pièces des îles Australes
(deux colliers et deux pointes de lance] acquises par le musée de Tahiti
et des Îles en 1980 après une complexe succession de propriétaires.
Ils se trouvaient jusque-là dans la collection de James Hooper (Phelps
1976 : $n^{0 s} 653,655,659-660 ;$ Millaud et Mu-Liepmann 2001), qui les
obtint à la suite d'un échange avec Kenneth Webster, lui-même les
ayant acquis auprès du musée de Leeds après la Seconde Guerre mon-
diale. Ils avaient auparavant appartenu à la Leeds Philosophical and
Literary Society, à qui Bennet les avait donnés aux alentours de 1829 -
1832. Bennet, quant à lui, les obtint probablement durant une visite à
Rurutu en septembre-octobre 1822 . II fit également don d'objets à la
Sheffield Literary and Philosophical Society, dont certains se trouvent
aujourd'hui au Cambridge University Museum of Archeaology and
Anthropology.

5. Difficilement traduisible en français, le terme agency est utilisé par Alfred Gell (1998) pour décrire l'action effective que certains objets, notamment les objets d'art, peuvent avoir sur leur entourage. Il est parfois traduit par intentionnalité, « agence » ou « agentivité », selon les contextes. (NdT)

6. La majorité des écrits sur l'iconoclasme se concentre sur la tradition occidentale. Parmi les études récentes, on compte celles de McClanan et Johnson (2005), de Besançon (2000) et de Freedberg (1989). Après la rédaction de ce texte, un article a été publié par Rambelli et Reinders (2007 : 21-27) ; il aborde les modes d'iconoclasme, et fait référence à la préservation et au rôle des musées. Les auteurs utilisent une expression semblable à celle utilisée dans ce texte, iconoclastic preservation [《préservation iconoclaste », NdT]. Ceci montre que la réflexion sur ce sujet est suffisamment avancée pour envisager des études transculturelles et permet de comprendre comment les images religieuses sont culturellement redéfinies si elles sont déplacées et re-présentées. 
de désacralisation de type iconoclaste. Dans ce cas comme dans d'autres, les idoles étaient dépouillées de leurs enveloppes et perdaient leurs pouvoirs en étant exposées dans des circonstances inappropriées. Il semble que les missionnaires jugèrent de plus en plus fréquemment que la destruction de l'idolâtrie pouvait être obtenue non seulement par la destruction physique des idoles, mais également par un processus de préservation, désacralisation et neutralisation du pouvoir. Selon les cas, pour les Polynésiens convertis comme pour les missionnaires, l'iconoclasme pouvait avoir lieu non seulement par l'annihilation mais aussi par la constitution de trophées. En poursuivant l'analogie militaire, ces idoles désacralisées peuvent être perçues comme les équivalents des parties de corps humains qui, dans l'histoire de l'humanité, lors de conflits idéologiques, ont été fréquemment conservées en tant qu'incarnations visibles de victoires militaires (réelles ou supposées).

La préservation iconoclaste des idoles par les missionnaires de la LMS n'était pas seulement destinée à des rituels d'humiliation à bord de navires ou sur des chaires de chapelles. Il s'agissait également d'une stratégie visant à assurer la viabilité commerciale de l'organisation et l'obtention de fonds financiers. Les tensions et les inquiétudes au sujet du financement des missions revenaient de manière chronique entre les personnes présentes «sur le terrain » et les administrateurs des quartiers généraux de Sydney et de Londres. Les preuves de succès sous la forme d'idoles - des indicateurs de performance - devinrent des éléments clés dans ces relations internes à la LMS, ces idoles pouvant également être exposées au musée créé à Austin Friars, à Londres, en 1814 7 . Celui-ci contenait initialement des « curiosités » sous la forme de spécimens d'histoire naturelle et d'objets ethnographiques. La donation des idoles de la famille Pomaré (dont il est question plus loin) insuffla une nouvelle orientation à la collecte, conduisant à l'exposition en Europe d'exemples d'idolâtrie païenne afin d'encourager les donateurs à soutenir financièrement les œuvres d'évangélisation. Les idoles capturées prouvaient que la conversion des païens était possible, tandis que le talent avec lequel les spécimens ethnographiques étaient réalisés montrait que ces mêmes païens étaient dignes des efforts civilisateurs des envoyés du christianisme.

Au cours des années 1820, le musée de la LMS prit une orientation quelque peu différente de celle des musées Leverian ou Bullock à Londres, ouverts avant lui, qui étaient des entreprises commerciales visant principalement à satisfaire la curiosité du public pour des merveilles « naturelles » et « artificielles ». Le musée de la LMS devint l'instrument essentiel de l'entreprise d'évangélisation qui transformait les charmants habitants d'une Arcadie des mers du Sud en des païens égarés devant être sauvés de toute urgence. Ce salut requérait des fonds réguliers et la fonction principale du musée, comme celle des expositions itinérantes organisées par les missionnaires au xIX ${ }^{e}$ siècle, était alors d'encourager les dons afin de financer ces missions en des terres lointaines. La conception que pouvaient avoir les Polynésiens du musée nous paraît peu claire aujourd'hui, mais les actions du chef tahitien Pomaré semblent avoir eu une grande influence sur la manière dont les membres de la LMS résolurent de l'utiliser pour poursuivre leurs objectifs. Si l'on rend justice à ces preuves, on peut suggérer que le Polynésien Pomaré fut à la source de l'orientation prise par le musée et des habitudes de collecte énergique adoptées par les membres de la mission afin de le remplir de trophées.

\section{Les idoles de Pomaré et un fare atua}

En 1816, Pomaré, converti depuis peu, donna quelques «idoles familiales » et écrivit dans une lettre fréquemment citée dans les publications des missionnaires: "Si vous le jugez approprié, vous pouvez les brûler toutes; ou, si vous le voulez, envoyez-les dans votre pays pour être inspectées par les peuples d'Europe afin qu'ils puissent satisfaire leur curiosité et connaître les dieux ineptes de Tahiti " (Missionary Sketches III, $1818: 3$ ). Les missionnaires choisirent la seconde option et ces idoles furent envoyées en Angleterre et reproduites par la LMS dans leur troisième brochure, intitulée Missionary Sketches, en 1818 (fig. 1). Par la suite, ces idoles, ainsi que la lettre de Pomaré, occupèrent une place centrale au musée de la LMS. Dans l'introduction du catalogue du musée publié en 1826, les directeurs indiquaient qu'ils exposaient les «trophées du christianisme » dans le but de «se conformer aux vœux du défunt roi de Tahiti » (anonyme 1826 : iv). Les idoles de la famille Pomaré semblent ainsi avoir été les premiers objets religieux que la LMS envoya de Polynésie vers l'Angleterre. Durant les décennies suivantes, les populations des îles de la Société, des îles Australes, des îles Cook et d'autres endroits de Polynésie donnèrent bien davantage encore aux missionnaires.

7. L'importance du rôle de ce musée dans les missions des mers du Sud a été abordée par Sivasundaram (2005). Gunson (1978) s'intéresse aux missionnaires de la London Missionary Society. 
Quelques-unes des idoles de Pomaré peuvent être aujourd'hui identifiées parmi les objets présents au British Museum. Les numéros 7 à 9 de la figure 1 correspondent probablement à la figure to'o aujourd'hui inventoriée comme LMS 102 (fig. 10), au manche de chassemouche en os de baleine LMS 57 et à la petite figure LMS 98 (Hooper $2006: \mathrm{n}^{\circ \mathrm{s}} 132,169$ et 128). En ce qui concerne LMS 98 , cette figurine de $10,5 \mathrm{~cm}$ de haut a été longtemps associée à un important fare atua (« maison du dieu ", fig. 2), lui aussi dans la collection LMS du British Museum (LMS 12O). Les deux éditions du Handbook du British Museum $(1910: 159 ; 1925$ : 164) montrent cette figure en compagnie du fare atua, où ce dernier est appelé « arche pour la figure d'une déesse ». Toutefois, cette figure, parmi les idoles de la famille Pomaré données aux missionnaires de la LMS en 1816, n'a pas pu appartenir à ce fare atua particulier, dont nous savons qu'il fut acquis par George Bennet en 1823.

Les circonstances dans lesquelles le fare atua fut obtenu nous donnent un aperçu des réactions complexes vis-à-vis du christianisme à Tahiti à cette époque. Bennet était à Papara, Tahiti, le $1^{\text {er }}$ octobre 1823. Dans les rapports officiels de la députation de la LMS, publiés à partir de documents originaux de James Montgomery, on trouve le passage suivant, probablement écrit par Daniel Tyerman :

$1^{\mathrm{er}}$ oct. [1823] Mr Bennet a obtenu un fare atua, ou maison d'un dieu, la seule relique de ce type que nous ayons vue dans ces îles; la destruction de tels objets fut presque totale, alors que les idoles elles-mêmes étaient préservées pour être transportées en Angleterre comme trophées du triomphe des Évangiles. Cette arche a été faite d'un seul bloc de bois ; sa forme est semblable à celle des maisons d'habitation, avec un toit pentu, et longue de trois pieds. Au-dessous se trouvait un trou cylindrique, muni d'une porte qui en fermait parfaitement l'ouverture. Il s'agissait du lieu de repos de l'idole. Le tout était supporté par quatre courtes pattes ressemblant à celles d'une tortue. L'idole elle-même semblait être d'une grande ancienneté - un diable femelle, hideusement informe, imitant l'humanité. Son nom était Tii Vahine, et on nous indiqua qu'elle avait massacré ses milliers [sic], et avait été hautement vénérée et adorée depuis des temps immémoriaux. Au moment du rejet général de l'idolâtrie, cette image et la maison dans laquelle elle était conservée furent mises au secret par quelques-uns de ses prêtres, dans une caverne au cœur des montagnes, et ne fut exhibée que plus tard, lorsque le tout fut apporté au marché et vendu, non pour sa valeur monétaire, mais comme curiosité (Montgomery 1831 : vol. II, 58).

La description très précise de ce passage se réfère indubitablement au fare atua conserve aujourd'hui au British Museum. On sait que la figure qui fut acquise en même temps qu'elle - appelée Tii Vahine (ti'i vahine, « image femme ")- n'est pas celle qui lui fut plus tard associée (LMS 98). Ainsi la localisation de la Tii Vahine demeuret-elle incertaine, bien qu'on puisse présumer que sa taille lui permettait d'être introduite dans l'ouverture cylindrique et qu'elle était anthropomorphe, « imitant l'humanité ». Une étude des figures féminines dont on attribue la collecte à Bennet pourrait révéler des candidates potentielles au nom de Tii Vahine. Il est même possible que ce nom ait été inscrit sur la figure même, puisqu'on connaît une image masculine du British Museum portant l'inscription « Ti'i Tane » (« image masculine » ou « image de Tane "; Hooper $2006: n^{\circ}$ 122). Apposer des inscriptions

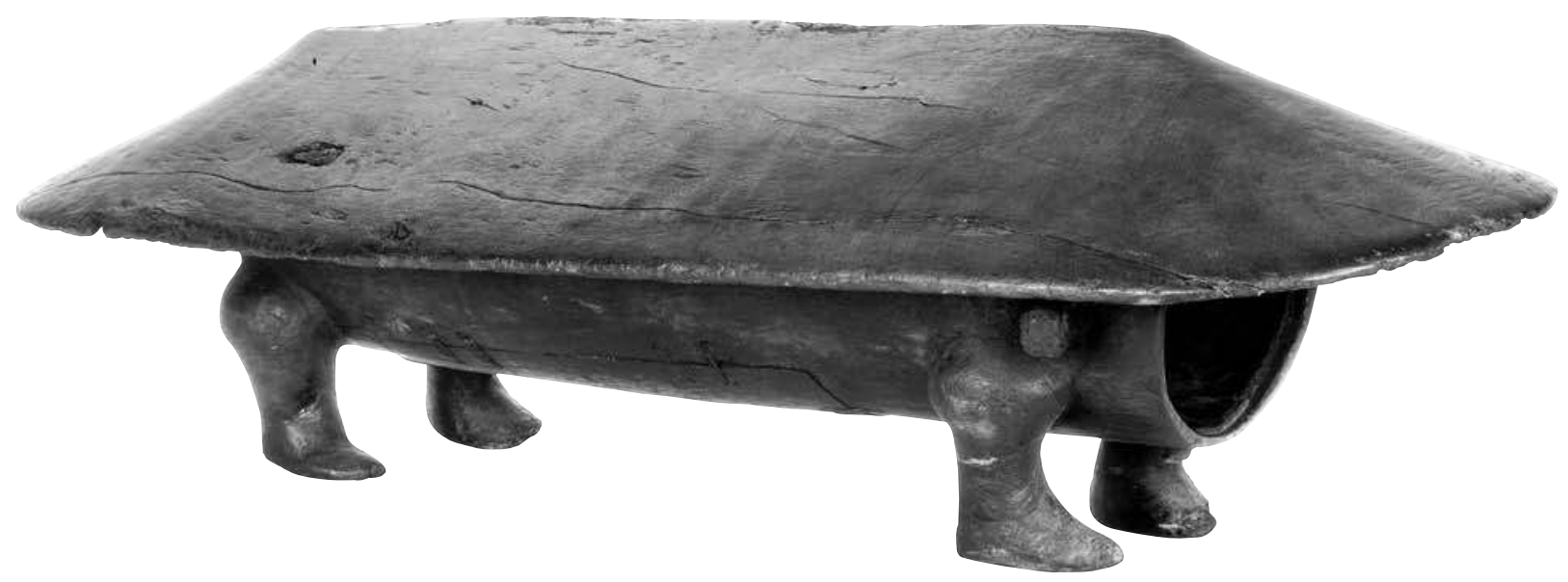

Fig. 2 Fare atua (maison du dieu, « arche "), Tahiti, îles de la Société. Bois et fibre de coco, $87 \mathrm{~cm}$. British Museum, LMS 120. @ The British Museum, Londres, courtesy Trustees of the British Museum. 


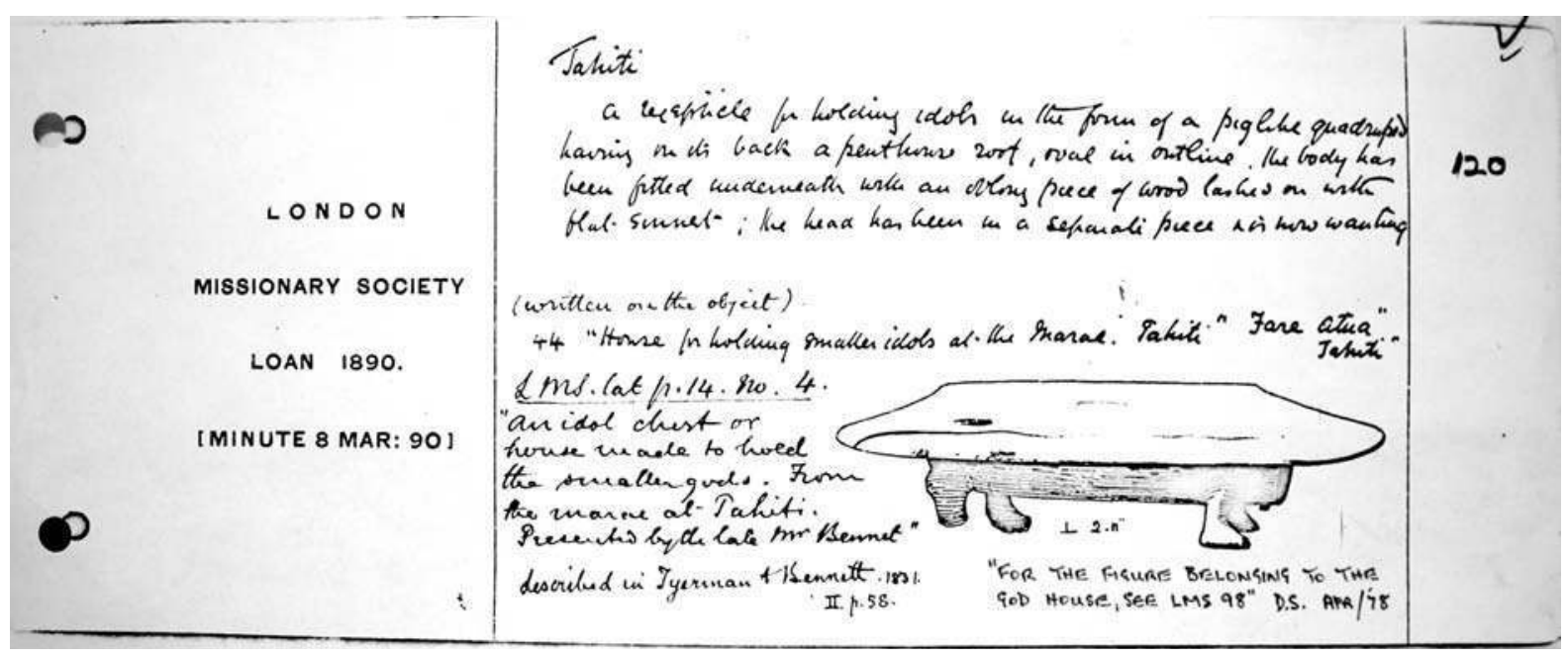

Fig. 3 Encart du catalogue du British Museum pour le fare atua, LMS 120, renvoyant à l'inscription sur le dessus. Photo Steven Hooper.

sur une image à des emplacements importants peut être considéré comme un acte iconoclaste et de désacralisation, sans même que cela soit intentionnel. Bennet écrivait directement à l'encre noire, d'une écriture large et ronde, sur certains objets, comme on peut le voir sur le toit de ce fare atua (fig. 3) et sur le dos, le front et les joues de l'image Tii Tane. Il écrivit aussi sur un bâton de bois du British Museum (O80.Oc.1075; auparavant l'âme d'un grand to'o) : «Oro, the God of War. Presented to G. Bennet by the widow Queen Pomare... ${ }^{8}$ " Pour ce qui est de l'image Tii Vahine acquise par Bennet avec le fare atua, les recherches montrent que tous deux ne formaient pas un ensemble parce qu'on sait désormais que ce fare atua fut originellement conçu pour recevoir un to'o, une image de cordelette et de plume associée au culte de 'Oro (Hooper 2005; Kaeppler 2007 : 98-102). Il est possible que la Tii Vahine de Bennet ait été une image to'o, mais la description ne semble pas correspondre.

La citation de Montgomery démontre que les objets comme le fare atua étaient déjà devenus rares vers 1823 . Bennet et Tyerman passèrent de nombreux mois aux îles de la Société entre 1821 et 1824. Le premier était un collectionneur avide, aussi était-il en bonne position pour pouvoir juger de ce qui était disponible. Bien sûr, comme le passage l'indique, de nombreux objets rituels furent cachés par ceux qui s'opposèrent à la christianisation et qui finirent par être vaincus durant la période de « rejet de l'idolâtrie » survenue à Tahiti en 1815-1816 (Davies 1961). À mesure que la résistance au christianisme diminuait et que les tentations du « marché » se faisaient plus fortes, quelques-uns de ces vieux objets réapparurent pour être vendus aux Européens - que les gens en aient été les propriétaires légitimes ou non. Nous ne savons pas ce que Bennet donna en échange du fare atua et de la Tii Vahine, mais il est établi qu'il disposait d'un stock de tissus européens, d'hameçons et autres objets en métal auxquels les Polynésiens accordaient alors une grande valeur.

Dans le passage cité, il existe une ambiguïté en ce qui concerne les références aux termes de "valeur » et de " curiosité ». Une distinction semble être faite entre la valeur du fare atua comme objet rituel important dans son cadre préchrétien et son intérêt en tant qu'antiquité dans son contexte chrétien. À cette époque, le terme « curiosité " n'avait pas de connotation péjorative, mais était appliqué à des choses faites avec talent et dignes d'intérêt. Il est probable que les missionnaires étaient conscients des changements de signification de ces objets qui, après avoir eu une valeur et un pouvoir spécifiques, devinrent par la suite des choses seulement dignes d'intérêt, du moins de leur point de vue. Ces deux aspects peuvent toujours s'appliquer à cet objet. Il s'agit en effet du seul exemple de ce type ; le talent avec lequel il a été réalisé se combine à ses formes puissantes pour transformer et déplacer son appréciation du plan de la simple curiosité à celui d'une œuvre d'art. Cette observation s'applique à d'autres objets de la région dont on verra à présent deux exemples, tout aussi uniques et remarquables.

8. « Oro, le Dieu de la Guerre. Présenté à G. Bennet par la Reine veuve Pomaré... » (NdT) 


\section{Les dieux-bâton de Rarotonga}

Il semble que les missionnaires n'aient pas été découragés par la taille des objets quant il s'agissait d'acquérir et de conserver ceux qu'ils jugeaient relever d'une signification particulière. L'immense dieu-bâton de Rarotonga des îles Cook (fig. 4), qui faisait partie des quatorze présentés aux missionnaires John Williams et Charles Pitman en 1827, en est un exemple. Williams, qui passa presque un an à Rarotonga en 1827-1828, fut le témoin de l'événement suivant, survenu peu de temps après son arrivée :

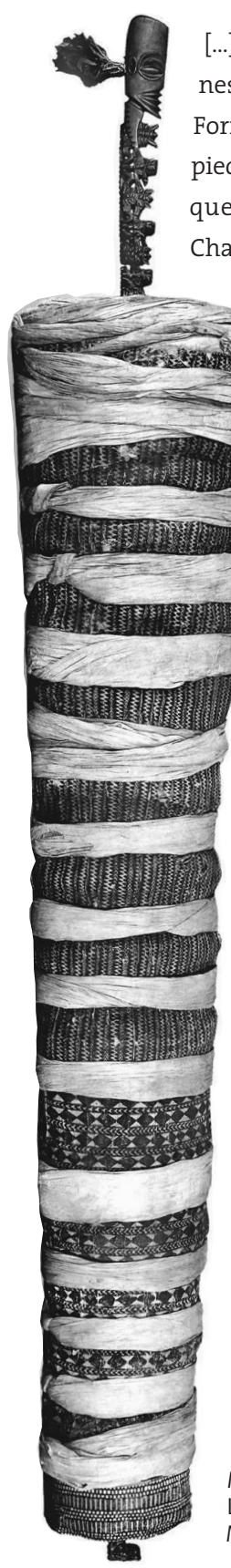

[...] nous observâmes un grand groupe de personnes venant vers nous, portant de lourds fardeaux. Formant une procession, ils vinrent déposer à nos pieds quatorze immenses idoles, la plus petite desquelles était à peu près longue de cinq yards. Chacune d'entre elles était composée d'une pièce d'aito, ou bois de fer, d'environ quatre pouces de diamètre, sculptée d'une imitation grossière de tête humaine à une extrémité et munie d'une figure obscène à l'autre, enroulée dans de l'étoffe indigène jusqu'à atteindre deux ou trois yards de circonférence. Auprès de la pièce de bois se trouvaient des plumes rouges et une enfilade de petites pièces de nacre polie provenant d'huîtres perlières, dont il est dit qu'elles sont le manava, ou âme du dieu. Certaines de ces idoles furent démembrées devant nos yeux, d'autres furent conservées afin de décorer les chevrons de la chapelle que nous nous proposions d'élever; et une fut gardée afin d'être envoyée en Angleterre et se trouve aujourd'hui au Missionary Museum. Son apparence n'est toutefois plus aussi respectable qu'en sa propre contrée ; car les officiers de sa Majesté britannique, craignant qu'elle ne fut utilisée pour frauder le Roi, la mirent en pièce sans cérémonie; et étant moins doués pour construire des dieux qu'ils ne le sont pour protéger les revenus royaux, ils ne la rendirent pas aussi belle que lorsqu'elle était un objet de vénération pour les gens égarés de Rarotonga (Williams 1837 : 115-116).

Fig. 4 Grand dieu-bâton, Rarotonga, îles Cook. Bois, étoffe d'écorce, plumes, $396 \times 65 \mathrm{~cm}$. British Museum, O78.Oc.845, () The British Museum, Londres, courtesy Trustees of the British Museum.

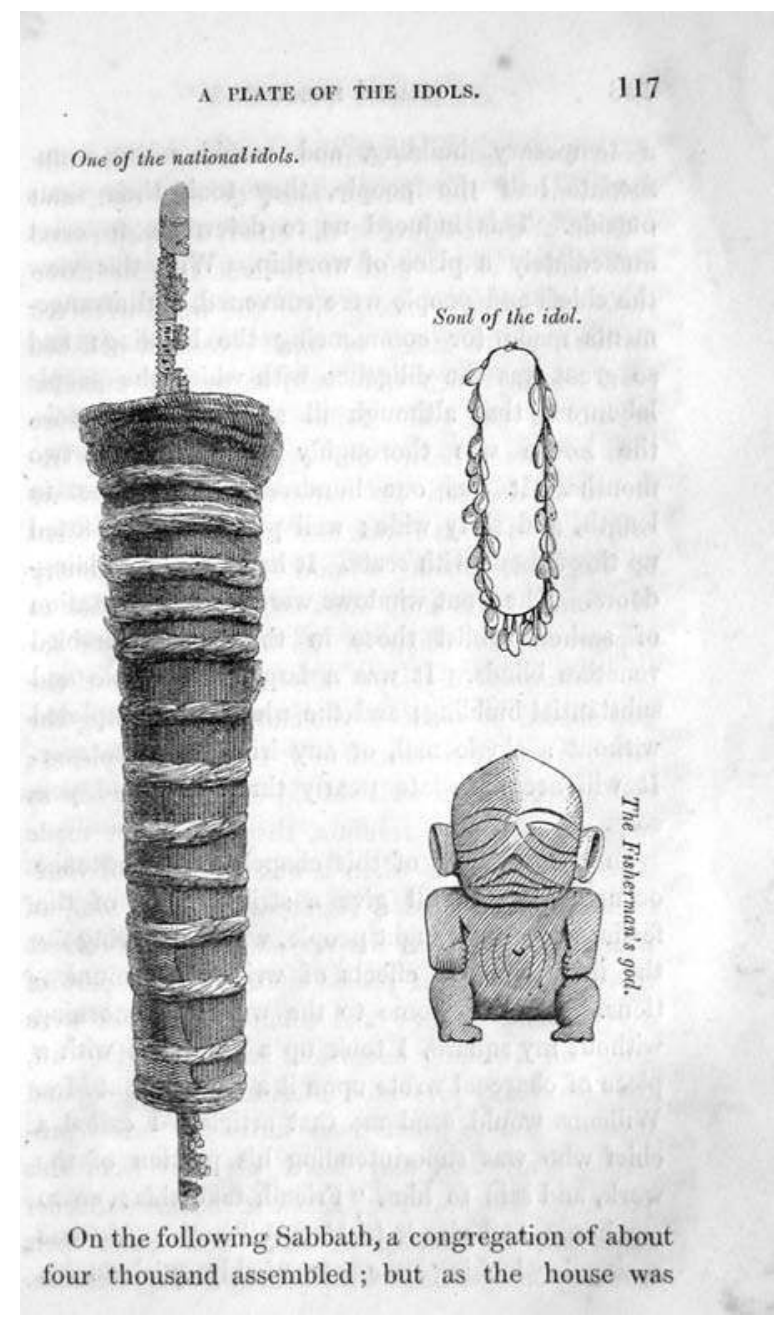

Fig. 5 Page 117 de Missionary Enterprises de John Williams (1837), montrant la tête du dieu-bâton émergeant de la partie la plus

épaisse du rouleau d'étoffe d'écorce. Photo Steven Hooper.

Parmi les nombreux points intéressants de ce passage, on trouve l'intention de décorer les chevrons de la chapelle chrétienne à l'aide des images des dieux vaincus, pratique fréquente dans l'activité d'évangélisation de la Polynésie. Il est intéressant de noter que, lorsque des images païennes étaient incorporées dans l'architecture des chapelles ou, comme cela pouvait aussi arriver, lorsque des temples païens étaient conservés mais consacrés au Christ, il s'agissait de donner à la population locale un moyen de comprendre et d'interpréter la nouvelle religion en des termes proches de leurs pratiques religieuses préexistantes.

De ces images " mises en pièces » ou placées dans les chevrons des chapelles, ont survécu un certain nombre d'extrémités sculptées, aujourd'hui dans diverses collections, dont la seule virtuellement intacte est celle mentionnée comme ayant été envoyée en Angleterre, 


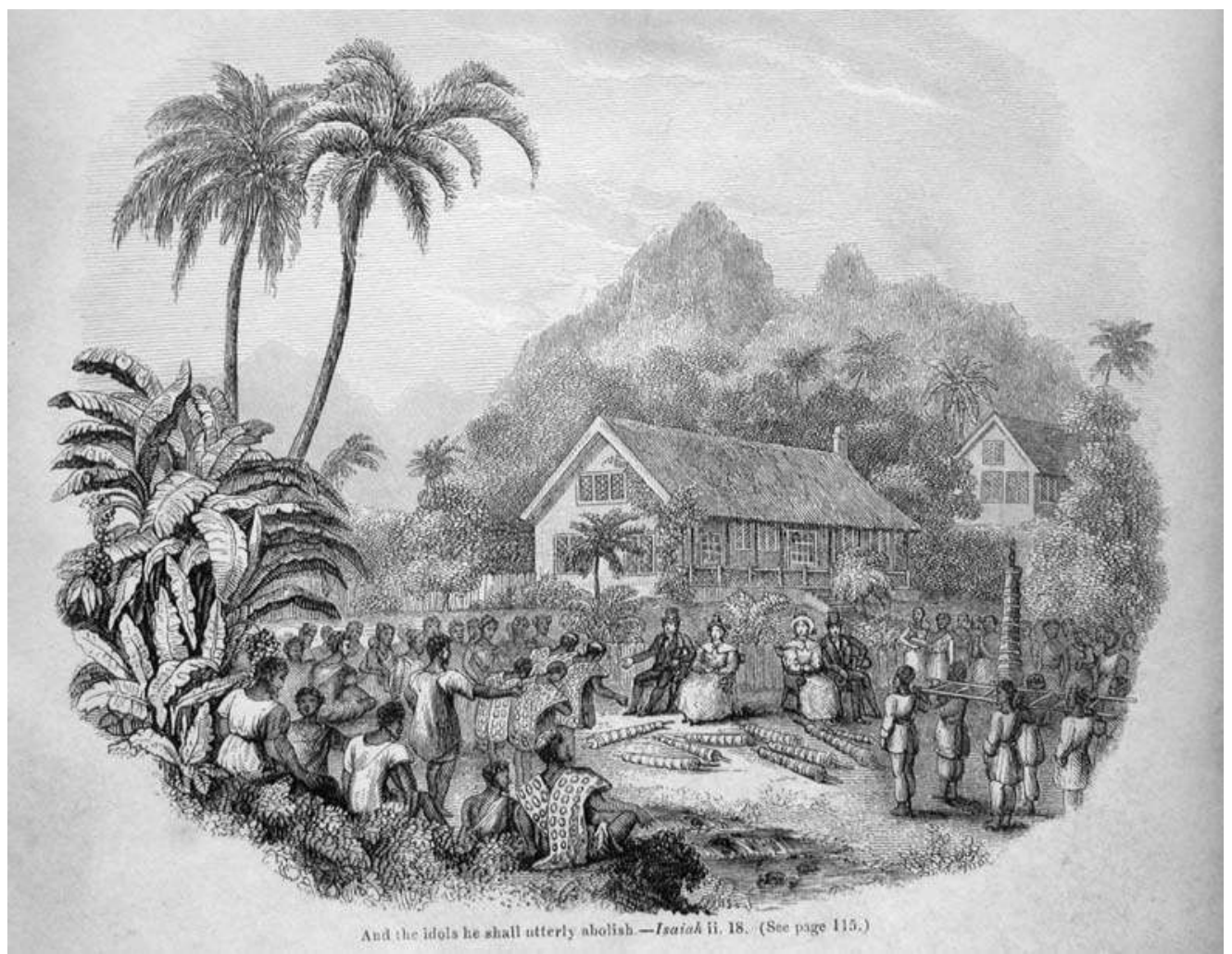

Fig. 6 Gravure de la page de titre de Missionary Enterprises de John Williams (1837), montrant une scène idéalisée de présentation d'idoles aux missionnaires Williams, Pitman et leurs épouses en 1827 à Rarotonga. Photo Steven Hooper.

aujourd'hui au British Museum (fig. 4). Comme le regrette Williams, les inspecteurs des douanes anglaises démembrèrent l'image pour y vérifier sans doute la présence de contrebande cachée dans l'étoffe, aussi l'arrangement précis de l'étoffe enroulée pourrait ne pas être tel qu'il l'était à Rarotonga. Une gravure de George Baxter publiée dans le livre de Williams, Missionary Enterprises (1837 : 117; fig. 5), pourrait s'inspirer d'un dessin réalisé à Rarotonga par Williams lui-même. En effet, le rendu des motifs de l'étoffe d'écorce est différent de celui de l'objet tel qu'on le connaît aujourd'hui - après l'intervention des officiers de sa Majesté britannique. Dans la gravure, la section inférieure gravée d'une « figure obscène ", que l'on sait par d'autres exemples avoir été un pénis représenté de manière naturaliste, n'est montrée que partiellement et sans son extrémité. Cette section inférieure du dieu-bâton a été coupée et manque aujourd'hui; une seule petite figure de profil a demeuré. À noter également le fait que, dans cette gravure, la section de la tête du bâton, qui fut aussi coupée plus tard (probablement pour en faciliter le transport), émerge de la partie la plus large du rouleau d'étoffe d'écorce. Il est probable qu'il s'agisse de sa position originale, mais d'autres illustrations anciennes la montrent émergeant du côté le plus étroit. Ainsi, une gravure sur la page de titre de Missionary Enterprises, qui sert d'illustration à la citation ci-dessus (fig. 6), montre une scène imaginaire de piété où les dieux-bâton sont déposés aux pieds des missionnaires Williams et Pitman et de leurs épouses. Il semble que Baxter ait fondé sa description des dieux-bâton sur l'exemple exposé au musée de la LMS à Londres, puisque la tête du dieu-bâton porté dressé se projette depuis l'extrémité étroite du rouleau d'étoffe d'écorce. Les officiers des douanes paraissent avoir non seulement dérangé l'étoffe d'écorce, mais également inversé les deux extrémités coupées du bâton - voire jeté l'extrémité inférieure phallique, bien que cela puisse être l'œuvre ultérieure de la LMS à Londres. Dans une gravure du musée de la LMS réalisée en 1859 (fig. 7), on peut voir que le dieu-bâton est exposé debout, sa tête émergeant de l'extrémité étroite de l'étoffe enroulée. Il est aussi dessiné selon une taille plus grande que nature, proportionnellement exagérée par l'échelle réduite de la taille des visiteurs comparée à la hauteur de la table. L'examen des deux extrémités coupées du bâton central, destiné à déterminer à quelle extrémité la tête 


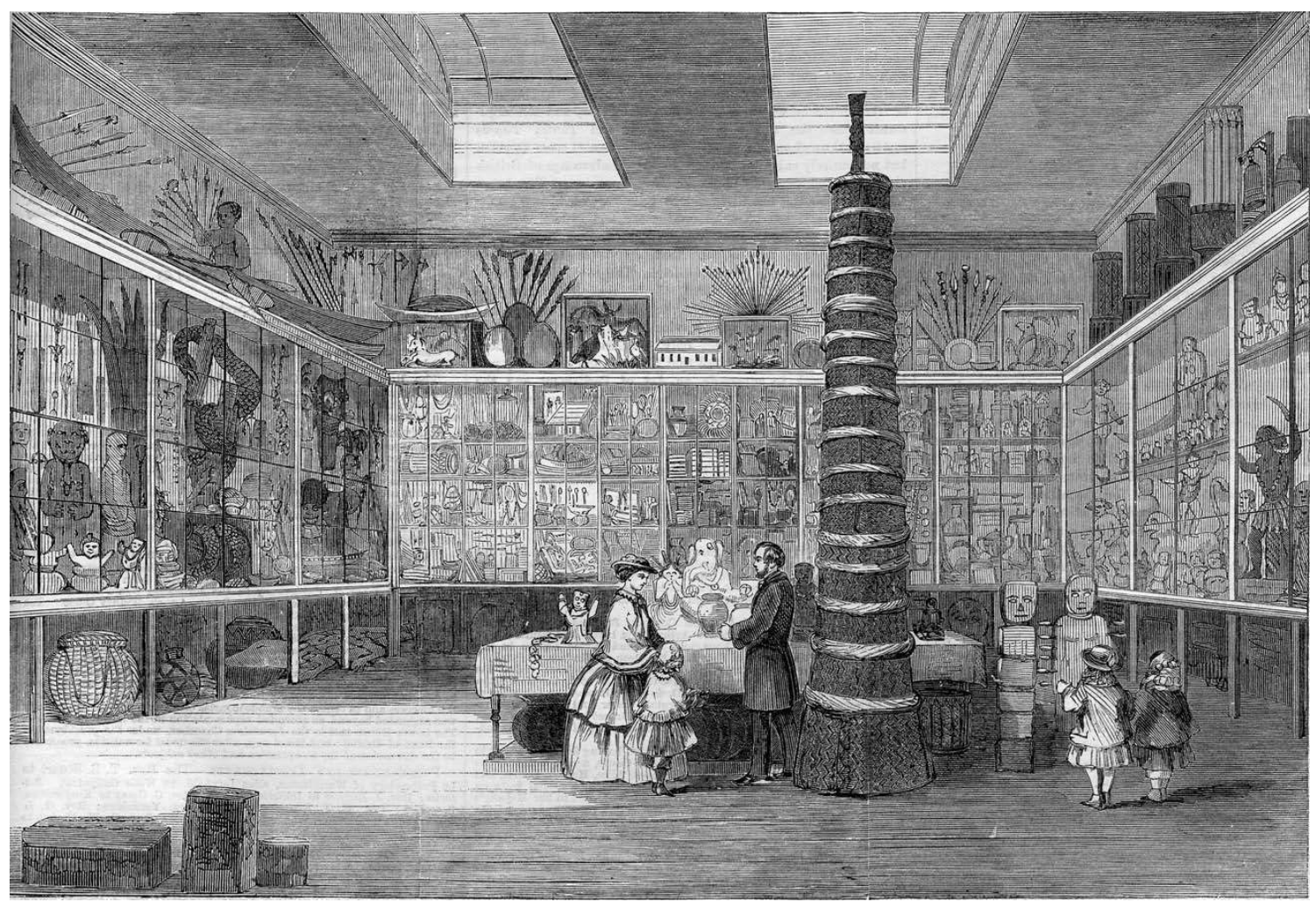

Fig. 7 Le musée de la London Missionary Society Illustrated London News, 25 juin 1859. D’après les archives de la London Missionary Society/Council for World Mission, Londres. Photo Glenn Ratcliffe.

pouvait appartenir, ne donne pas de résultat concluant, la taille et la forme des deux extrémités étant similaires. Le fait que celles-ci paraissent avoir été tranchées plutôt que sciées ne permet pas de distinguer d'ajustement précis. Du point de vue iconographique, la tête aurait pu être placée aux deux extrémités. En effet, une petite figure, orientée de profil, suit généralement la tête en séquence, mais une telle figure, orientée de manière appropriée, peut se retrouver aux deux extrémités coupées du bâton? 9

Ces grandes images de Rarotonga, appelées ki'iki'i, servaient vraisemblablement de réceptacles élaborés de la puissance divine, celle-ci se manifestant dans les matériaux qui les composent et dans la forme des sculptures. Les plumes et les coquillages polis contenus dans l'étoffe, aujourd'hui disparus, étaient clairement d'une grande importance, comme l'était la métaphore sexuelle du bâton de bois et de l'étoffe qui l'enveloppait, tous deux produits respectifs du travail des hommes et des femmes. Il s'agissait de matérialiser la relation complémentaire de ces deux composantes, symbolisant non seulement les deux sexes, mais également les chefs et le peuple. L'abondance et la vie sont issues des dieux, mais le maintien de relations complémentaires adé- quates est également un facteur clé pour obtenir la prospérité. En Polynésie, le rôle puissant de protection et de nutrition des femmes était célébré et symbolisé par l'enroulement dans des étoffes d'écorce et de nattes, pratique qui a toujours lieu dans certaines régions sur le corps des personnes à des moments clés de leur vie - de la naissance au mariage et à la mort.

Le processus de christianisation de Rarotonga ne fut pas aussi simple que décrit dans l'illustration de Missionary Enterprises (fig. 6). Après l'arrivée des pasteurs polynésiens en 1823, de nombreux conflits internes eurent lieu entre les factions chrétiennes et non chrétiennes. Il est probable que les quatorze idoles offertes à Williams et Pitman en 1827 par la faction chrétienne ont été capturées (peut-être dans une démarche de collecte iconoclaste) lors d'une attaque contre les non-chrétiens durant les deux années précédentes. La preuve en est donnée par le révérend Robert Bourne, qui se rendit à Rarotonga en 1825 et relata qu'il avait

9. Je suis reconnaissant envers le Dr Julie Adams, ancienne étudiante à la Sainsbury Research Unit et actuellement Research Assistant au British Museum, qui a coorganisé l'exposition Power and Taboo en 2006, pour m'avoir indiqué ces intéressants problèmes de position et en avoir discuté avec moi. 
vu quatorze idoles en possession des chrétiens (Sissons 2007 : 57-58; Maretu 1983 : 70-71). Il est fort possible qu'il s'agisse de celles qui furent présentées lors de la mise en scène dramatique de 1827 à Williams, nouvel arrivant, perçu par les habitants de Rarotonga comme le prêtre principal des missionnaires de la LMS. Cette utilisation stratégique des idoles capturées par les habitants de Rarotonga n'est pas explicite dans le texte de Williams, qui laisse penser que la présentation était un acte spontané de piété, à la suite de la conversion. En fait, la destruction des marae (temples) ou la confiscation des propriétés rituelles de valeur était une tactique employée durant les conflits interpolynésiens, et ceux qui présentèrent les dieuxbâton au missionnaires étaient probablement conscients de la manière dont cette tactique pouvait aider leur propre cause tout en nuisant à leurs adversaires locaux.

\section{Une figure-coffret de Rurutu}

Le dernier objet dont on discutera ici est peut-être la plus célèbre sculpture de l'ancienne Polynésie qui ait survécu. Il s'agit de la figure de Rurutu des îles Australes, aujourd'hui connue principalement sous le nom de $A^{\prime} a$ (fig. 8). Cet objet a été étudié ailleurs plus en détail, notamment du point de vue de sa probable utilisation originelle comme reliquaire, pour le crâne et les os longs d'un ancêtre révéré (Hooper 2007). Mais ce qui nous intéresse ici est la manière dont il fut acquis par la LMS. Comme pour les autres objets, les circonstances de son acquisition sont connues ; il est également visible dans la gravure de 1859 du musée de la LMS (fig. 7), figurant debout, au centre de la vitrine, sur l'extrême gauche de l'image. Cette sculpture fut remarquée pour la première fois en Europe en août 1821, lorsqu'elle fut apportée de Rurutu afin d'être présentée à John Williams et Lancelot Threlkeld, missionnaires de la LMS à Ra'iatea.

Comme le décrit Williams (1837: 38-43), suite à une épidémie qui dévasta Rurutu autour de 1818-1819,

un nommé Au'ura embarqua, au début de l'année 1821, en compagnie de sa femme et d'une trentaine de compagnons pour un voyage en pirogue, et toucha d'abord Tubuai. Durant le voyage du retour, une tempête les détourna et ils finirent par atteindre Ra'iatea le 8 mars, où il passèrent trois mois à se remettre de leurs épreuves. Les habitants de Rurutu connaissaient Ra'iatea et son grand marae Taputapuatea à Opoa, un des sites religieux les plus importants du Pacifique central. Dans l'esprit d'Au'ura, le fait que des prêtres de la nouvelle religion chrétienne aient installé leur propre

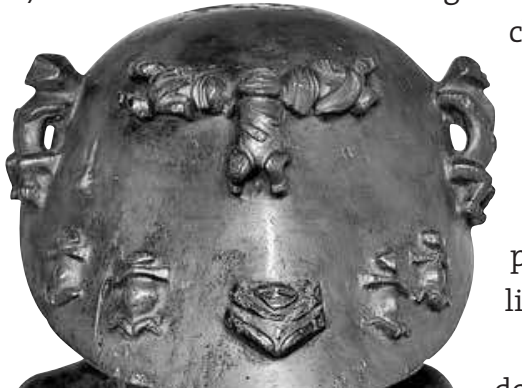
chapelle sur Ra'iatea revêtait une signification importante. Aussi Au'ura fit preuve d'un grand intérêt pour les missionnaires, ces derniers, à leur tour, voyant en lui un instrument potentiel pour leur œuvre d'évangélisation.

La plupart des voyageurs bloqués, dont Au'ura, furent renvoyés à Rurutu par le brick Hope en juillet 1821, accompagnés de deux diacres originaires de Ra'iatea nommés Mahamene et Puna, et de leurs épouses. Threlkeld y fit également remorquer son propre bateau. Selon Williams :

[9 août 1821] Après une absence d'un petit peu plus d'un mois, nous eûmes le plaisir de voir le bateau [celui de Threlkeld] revenir

[à Ra'iatea], chargé des trophées de la victoire, les dieux des païens capturés dans cette guerre non sanguinaire, vaincus par le pouvoir du Prince de la Paix... Une réunion se tint dans notre grande chapelle, pour communiquer la délicieuse nouvelle à notre peuple, et remercier Dieu pour avoir gracieusement couronné nos premiers efforts de diffusion de la connaissance de Son Nom... Durant la soirée, les idoles rejetées furent exposées publiquement depuis la chaire. Une en particulier, Aa, le dieu national de Rurutu, provoqua un intérêt considérable; car, en plus d'être orné de petits dieux sur son extérieur, une porte fut découverte dans son dos ; à son ouverture, il s'avéra être empli de petits dieux, et pas moins de vingt-quatre en furent sortis l'un après l'autre, et exposés à la vue du public. Il est dit être

Fig. 8 Figure-coffret (A'a), Rurutu, îles Australes. Bois, $117 \mathrm{~cm}$. The British Museum, LMS 19. (c) The British Museum, Londres, courtesy Trustees of the British Museum. l'ancêtre par qui leur île fut peuplée et qui fut déifié après sa mort (Williams 1837 : 43-44). 
L'acquisition des «idoles rejetées " était un coup d'éclat pour les missionnaires, mais c'était également une stratégie pour éviter qu'elles ne soient brûlées. Dans une lettre datée du 4 juillet 1821 au révérend George Burder à la LMS à Londres, Threlkeld écrit que le capitaine Grimes du Hope allait partir pour Rurutu :

Mon bateau part avec le navire et nous avons envoyé deux natifs et leurs femmes qui doivent enseigner les Rurutains \& [sic] pour lire nous leur avons fourni des livres... le chef [Au'ura] vous promet leurs dieux nous vous recommandons de ne pas les brûler mais de les envoyer comme captifs à la Société... Nous envoyons un bon bateau [un équipage] pour ramener mon bateau à bon port, s'ils parviennent à en trouver le chemin ${ }^{10}$.

En définitive, le capitaine Grimes quitta Rurutu peu de temps après avoir déposé ses passagers, non sans avoir tout d'abord acquis une idole auprès d'Au'ura (Hooper 2007: 164-165). Les autres " prisonniers » durent attendre le bateau de Threlkeld pour être envoyés à Ra'iatea.

Selon les écrits des pasteurs de Ra'iatea, une réunion eut lieu après le départ de Grimes de Rurutu pour annoncer la bonne nouvelle des Évangiles, ainsi que l'intention qu'avaient les chrétiens fraîchement arrivés de transgresser certains tabous, notamment ceux relatifs aux lieux sacrés et à la consommation de porc et de tortue par les femmes en compagnie des hommes. Les prêtres locaux menacèrent de punition divine ceux qui oseraient se comporter de telle manière. Leur crédibilité, tout comme celle des dieux qu'ils représentaient, fut mise à l'épreuve. Le fait qu'aucun effet nuisible ne fût observé chez ceux qui transgressèrent les tabous conduisit apparemment au succès du groupe de chrétiens et à la défaite des prêtres païens. Ces derniers, gardiens d'un important équipement rituel comprenant les idoles, furent apparemment contraints de les rendre après leur humiliation. Comme c'est probable, si A'a était l'objet rituel le plus important de Rurutu à cette époque, cette image de dieu ancestral était donc à la fois le centre des rituels préchrétiens et la cible majeure de l'iconoclasme postchrétien. Au'ura et les autres chrétiens locaux engagés dans cette guerre avaient pour directive de ne pas détruire mais de faire des « prisonniers ». Ainsi, bien que les marae aient été désacralisés et les images sans doute détruites durant les premiers élans zélés de conversion, de nombreux autres objets, incluant $A^{\prime} a$, furent préservés pour être transférés à Ra'iatea. De là, ils trouvèrent finalement leur chemin jusqu'au musée de Londres et dans les publications de la LMS (fig. 9).
Une telle séquence d'événements n'était pas contraire aux pratiques guerrières indigènes. Une des principales tactiques de guerre polynésienne était de perturber le cycle rituel d'offrandes et de sacrifices des ennemis, affectant ainsi la relation de soutien mutuelle établie avec leurs dieux et, par là même, les affaiblissant. Cette perturbation était obtenue en détruisant les récoltes et le bétail (les moyens essentiels aux sacrifices de nourriture), et en profanant les sites et les propriétés sacrés. Ceux qui étaient tués ou faits prisonniers, de même que les objets rituels importants, se trouvaient insérés dans le cycle sacrificiel productif des vainqueurs, utilisés durant des rituels publics qui célébraient le pouvoir de leurs propres divinités en humiliant l'ennemi et par conséquent ses dieux. Dès lors, le procédé de l'exposition de $A^{\prime} a$ et des autres idoles dans la chapelle de Ra'iatea ne serait pas apparu comme totalement étranger à un public polynésien.

\section{Conclusion}

Ce tour d'horizon de quelques-uns des objets polynésiens les plus importants acquis par la LMS a permis de montrer que la collecte comme forme d'iconoclasme était une tactique des missionnaires, à des fins apparemment contradictoires mais néanmoins complémentaires. Les motivations des insulaires et des missionnaires étaient complexes et s'adaptaient à des circonstances changeantes. Ainsi, les Polynésiens étaient-ils autant impliqués que les Européens dans la capture d'idoles, chacun tentant de tirer avantage de cette période pleine d'incertitudes ${ }^{11}$. Il apparaît également que les Polynésiens s'impliquaient de près dans les décisions stratégiques relatives à la destruction et à la préservation. Les actions

10. Les références aux lettres non publiées renvoient à des documents conservés dans les collections du Council for World Mission (CWM) à la bibliothèque de la School of Oriental and African Studies (SOAS), université de Londres : South Seas/Incoming Correspondence, Box 3B. Je tiens à exprimer ma reconnaissance envers Geoffrey Roper, de la SOAS, qui m'a fourni les photocopies des documents durant la fermeture temporaire des archives du CWM au début de 2007. Comme c'est le cas pour les dieux-bâton de Rarotonga, le récit relatif à A'a donne une image assez directe du processus de conversion - les Polynésiens « voyant la lumière » et déposant leurs idoles. Toutefois, les archives des correspondances révèlent une situation plus complexe, impliquant les pasteurs polynésiens, les rivalités locales, les disputes et les mouvements stratégiques dans lesquels les missionnaires britanniques sont soit absents, soit des spectateurs peu informés.

11. Jukka Siikala a fait le point sur la situation religieuse et politique aux îles de la Société durant cette période ( 1982 : 207-249), en insistant sur le lien entre le contrôle des idoles par les chefs et le pouvoir temporel. 


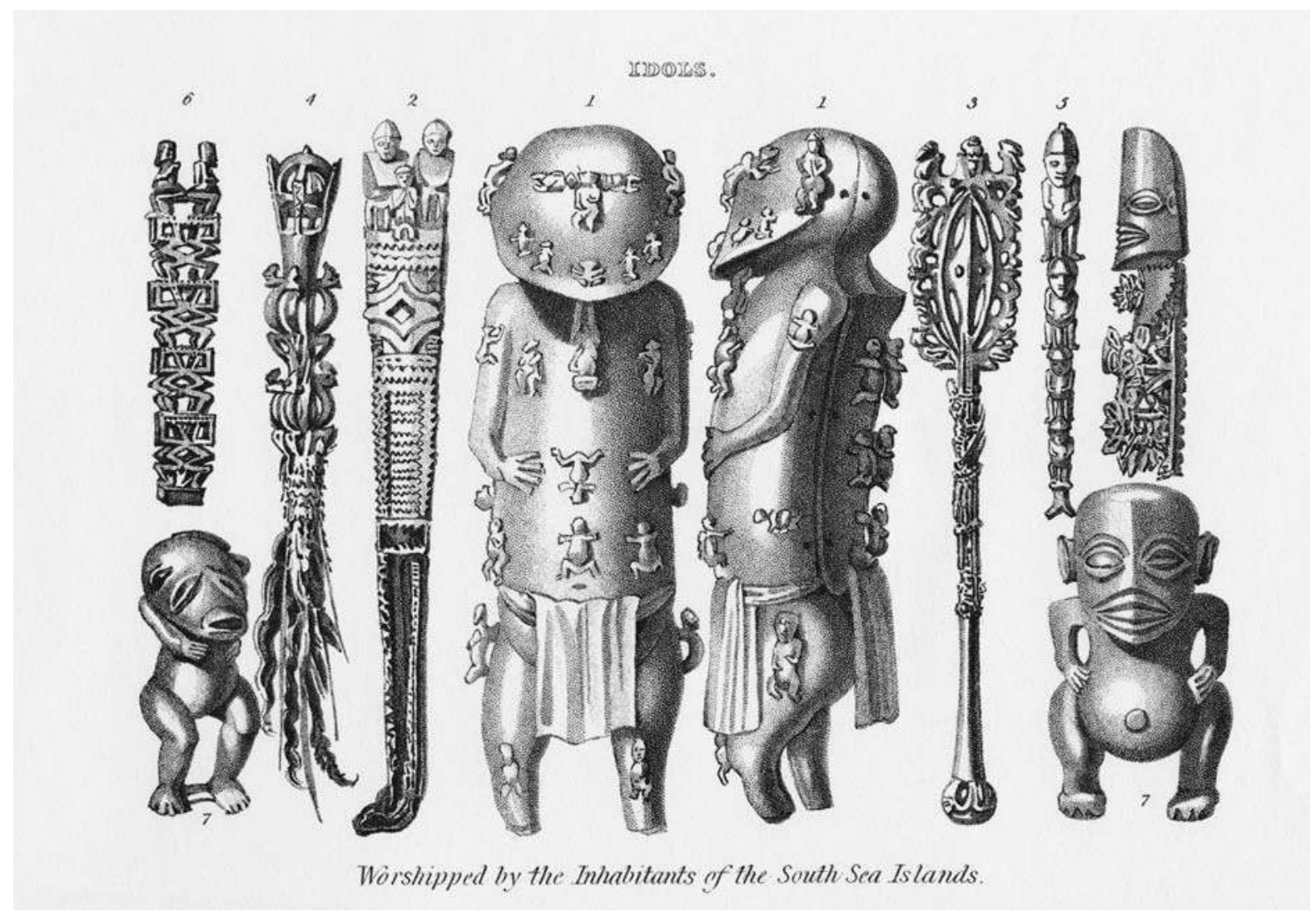

Fig. 9 Frontispice du volume II de Polynesian Researches de William Ellis (1829) montrant un ensemble d'idoles, dont A'a, des îles de la Société, des îles Australes et des îles Cook. Photo Steven Hooper.

de Pomaré lui-même concernant ses «idoles familiales " semblent avoir eu une influence majeure sur le développement du musée de la LMS à Londres. Celle-ci exploita sans aucun doute sa conversion au travers de ses publications, tandis que Pomaré suivait également ses propres intérêts stratégiques en se convertissant au christianisme et en rendant ses idoles. Les missionnaires finirent par le comprendre, comme en témoigne cette remarque de Lancelot Threlkeld dans une lettre du 8 juillet 1822, écrite après la mort de Pomaré survenue l'année précédente : « Son vrai but était de se saisir de toutes les îles sous le faux-semblant de les christianiser. »

Bien que nous puissions aujourd'hui déplorer le destin de tant d'objets importants durant cette période, résultat d'un iconoclasme par destruction, il faut reconnaître à cette politique d' "iconoclasme par collection" d'avoir permis la survie d'autres objets considérés comme un digne héritage. Presque deux siècles plus tard, ces derniers sont désormais le sujet de nouvelles appréciations, évaluations et réévaluations, pour les Polynésiens comme pour les Européens. En continuant à incarner les ancêtres, ils prennent également leur place parmi les grandes traditions artistiques mondiales.

mots clés / keywords : collection // collecting • iconoclasme // iconoclasm • missionnaires // missionaries • Polynésie // Polynesia • musées // museums • trophées // trophies • idoles // idols.

Sainsbury Research Unit Sainsbury Centre for Visual Arts, Université d'East Anglia S.Hooper@uea.ac.uk

Traduction de l'anglais par Ludovic Coupaye.

Cet article se fonde sur des recherches effectuées dans le cadre du projet Polynesian Visual Arts: meanings and histories in Pacific and European cultural contexts, financé par le Arts and Humanities Research Council (AHRC, bourse n RG/AN10253/APN16516), dont le soutien a été précieux. Je remercie également Jill Hasell, Lissant Bolton et Jenny Newell pour avoir facilité l'accès aux collections du British Museum, et Maia Jessop, Karen Jacobs et Julie Adams pour les discussions autour de cet article. 


\section{Bibliographie}

\section{ANONYME}

1818 "The Family Idols of Pomare", Missionary Sketches, III, octobre. Londres, London Missionary Society.

1826 Catalogue of the Missionary Museum, Austin Friars; Including Specimens in Natural History, Various Idols of Heathen Nations... Londres, W. Phillips.

\section{BESANÇON, Alain}

2000 The Forbidden Image: an intellectual history of iconoclasm. Londres, University of Chicago Press; traduction de L'Image interdite : une histoire intellectuelle de l'iconoclasme. Paris, Fayard, 1994.

\section{Borofsky, Robert et Howard, Alan} 1989 « The Early Contact Period», in Alan Howard et Robert Borofsky [éd.], Developments in Polynesian Ethnology. Honolulu, University of Hawaii Press : 241-275.

\section{BRITISH MUSEUM}

1910 Handbook to the Ethnographical Collections. Oxford, Oxford University Press for the Trustees of the British Museum.

1925 Handbook to the Ethnographical Collections, $2^{\mathrm{e}}$ éd. Oxford, Oxford University Press for the Trustees of the British Museum.

DAVIES, John

1961 The History of the Tahitian Mission, 1799-1830: with supplementary papers from the correspondence of the missionaries, Colin Newbury (éd.). Cambridge, Cambridge University Press for the Hakluyt Society.

\section{ELLIS, William}

1829 Polynesian Researches, during a residence of nearly six years in the South Sea Islands, 2 vol. Londres, Fisher, Son and Jackson.

\section{FREEDBERG, David}

1989 The Power of Images: studies in the history and theory of response. Chicago, University of Chicago Press.

\section{GeLL, Alfred}

1998 Art and Agency. An Anthropological Theory. Oxford, Clarendon Press.

Gunson, Niel

1978 Messengers of Grace: evangelical missionaries in the South Seas. Melbourne, Oxford University Press.

\section{Hooper, Steven}

2005 « On Looking at a Tahitian God-house », Journal of Museum Ethnography 17 : 89-100.

2006 Pacific Encounters: Art \& Divinity in Polynesia 1760-1860. Londres, British Museum Press.

2007 «Embodying Divinity: the Life of A'a », in Steven Hooper (éd.), Polynesian art: histories and meanings in cultural contexts, numéro spécial, The Journal of the Polynesian Society 116(2) : 131-179.

2008 Polynésie : arts et divinités 1760-1860. Paris, Réunion des musées nationaux-musée du quai Branly.

Humphrey, Caroline et Hugh-JONES, Stephen (éd.)

1992 Barter, Exchange and Value: an anthropological approach. Cambridge, University of Cambridge Press.

\section{JACOBS, Karen}

À paraître « Mapping the George Bennet Collection: the cartography of collecting ».

\section{Kaeppler, Adrienne}

2007 «Containers of Divinity », in Steven Hooper [éd.], Polynesian art: histories and meanings in cultural contexts, numéro spécial, The Journal of the Polynesian Society 116[2] : 97-130.

\section{LAVONDÈs, Anne}

1996 « L'histoire de A'a de Rurutu et l'évolution des mythes », in Michèle Julien et al (éd.), Mémoire de Pierre, mémoire d'Homme : tradition et archéologie en Océanie. Hommage à José Garanger. Paris, Publications de la Sorbonne («Homme et Société » 23) : 313-331.

\section{MARETU}

1983 Cannibals and Converts: radical change in the Cook Islands. Suva, Institute of Pacific Studies, University of the South Pacific-Ministry of Education, Rarotonga.

McClanAn, Anne et JoHnson, Jeff (éd.)

2005 Negating the Image: case studies in iconoclasm. Aldershot, Ashgate.
Millaud, Hiriata et Mu-Liepmann, Véronique (éd.)

2001 Les Collections : musée de Tahiti et des Îles - Te Fare lamanaha. Papeete, musée de Tahiti et des Îles-Te Fare lamanaha.

\section{Montgomery, James}

1831 Journal of Voyages and Travels by the Rev. Daniel Tyerman and George Bennet Esq. in the South Sea Islands 1821-1829, 2 vol. Londres, F. Westley and A.H. Davis.

\section{Phelps, Steven [Hooper, Steven]}

1976 Art and Artefacts of the Pacific, Africa and the Americas: the James Hooper Collection. Londres, Hutchinson.

\section{RAMBELLI, Fabio et ReINDERS, Eric} 2007 « What does iconoclasm create? What does preservation destroy? Reflections on iconoclasm in East Asia », in Stacy Boldrick et Richard Clay [éd.), Iconoclasm: contested objects, contested terms. Aldershot, Ashgate : 15-33.

\section{SAHLIns, Marshall}

1974 « On the Sociology of Primitive Exchange », in Marshall Sahlins, Stone Age Economics. Londres, Tavistock : 185-275.

\section{SiIKALA, Jukka}

1982 Cult and Conflict in Tropical Polynesia: a study of traditional religion, Christianity and nativistic movements. Helsinki, Academia Scientarium Fennica.

\section{Sissons, Jeffrey}

2007 « From Post to Pillar: god-houses and social fields in nineteenth-century Rarotonga », Journal of Material Culture 12(1) : 47-63.

\section{Sivasundaram, Sujit}

2005 Nature and the Godly Empire: Science and Evangelical Mission in the Pacific, 1795-1850. Cambridge, Cambridge University Press.

THOMAs, Nicholas

1991 Entangled objects: exchange, material culture and colonialism in the Pacific. Cambridge et Londres, Harvard University Press.

WiLLIAMs, John

1837 A Narrative of Missionary Enterprises in the South Sea Islands. Londres, John Snow. 


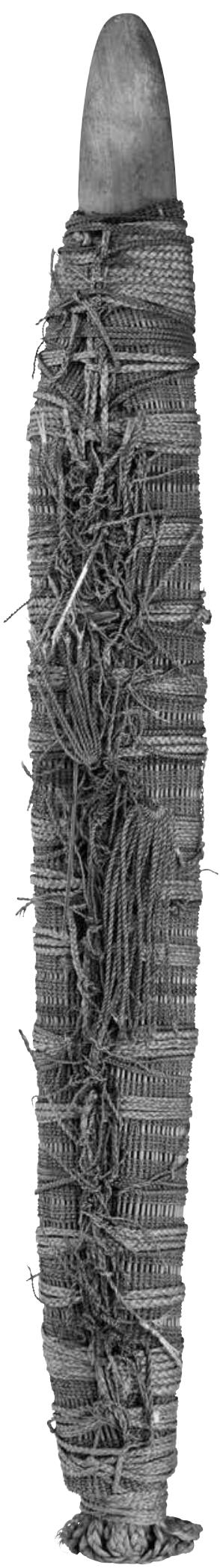

Fig. 10 Image de divinité, îles de la Société, The British Museum, LMS 102. @ The British Museum, Londres, courtesy Trustees of the British Museum. II s'agit très certainement du même objet que celui reproduit en $n^{\circ} 7$ dans la gravure de la fig. 1.

\section{Résumé / Abstract}

Steven Hooper, La collecte comme iconoclasme. La London Missionary Society en Polynésie. - Durant la première moitié du XIXe siècle, de nombreux objets importants de Polynésie furent collectés par la London Missionary Society et exposés dans son musée de Londres. Plusieurs sont abordés dans cet article. L'acquisition de ces objets, souvent référencés comme des idoles, était un processus plus complexe qu'on l'a souvent imaginé, qui impliquait des choix stratégiques pour les Polynésiens comme pour les missionnaires. On suggère que la préservation de ces objets a été faite sous l'émulation du chef tahitien Pomaré en 1816, et que différents types de pratiques iconoclastes eurent lieu, depuis l'iconoclasme par destruction jusqu'à l'iconoclasme par neutralisation et préservation. Polynésiens et missionnaires avaient des raisons évidentes de préférer l'iconoclasme par préservation, les premiers dans le but d'obtenir des trophées de leurs victoires sur leurs rivaux et de fournir des preuves matérielles de leur conversion, les seconds afin de fournir des preuves matérielles des succès de leur évangélisation et d'obtenir des fonds à travers les expositions au musée de la London Missionary Society. Ces opérations permirent la survie d'objets aujourd'hui réévalués comme une part importante du patrimoine et comme des œuvres d'art majeures.

Steven Hooper, Collecting as Iconoclasm. The London Missionary Society in Polynesia. - In the first half of the 19th century many important religious objects from Polynesia were collected by the London Missionary Society and displayed in their museum in London. Several are discussed in this paper. The acquisition of these objects, often referred to as idols, was a more complex process than often imagined, and one which involved strategic choices by Polynesians as well as missionaries. It is proposed that the preservation of these objects was stimulated by the Tahitian chief Pomare in 1816, and that different kinds of iconoclastic practice were enacted, from iconoclasm by annihilation to iconoclasm by disempowerment and preservation. Polynesians and missionaries alike had clear motives to enact iconoclasm by preservation, the former to obtain trophies from rivals and supply material tokens of conversion, the latter to provide evidence of evangelical success and a means of raising funds through exhibition in the London Missionary Society museum. These actions resulted in the survival of objects that are now being re-evaluated as important items of heritage and great works of art. 